\title{
Current-Voltage Characteristic of a Partially Ionized Plasma in Cylindrical Geometry
}

\author{
by \\ Joel L. Lebowitz and Alexander Rokhlenko \\ Department of Mathematics \\ Hill Center, Busch Campus \\ Rutgers University \\ New Brunswick, NJ 08903
}

\begin{abstract}
The properties of a partially ionized plasma in a long cylindrical tube subject to a uniform axial electric field are investigated. The plasma is maintained by an external ionizing source balanced by bulk and surface recombinations. Collisions between neutrals, whose density greatly exceeds the density of charged particles, and of neutrals with ions are sufficiently effective for their velocity distribution to be close to a Maxwellian with the same uniform temperature, independent of the external field. The behavior of the plasma is described by a collisional two-fluid scheme with charge neutrality in the interior of the tube. Approximate nonlinear equations for the hydrodynamical moments are obtained from a Boltzmann equation in which electron-neutral, electron-ion and electron-electron collisions are all important. It is found that under certain circumstances the current, and the temperature of the electrons undergo a drastic change, with hysteresis, as the electric field is varied.
\end{abstract}

PACS codes: 52.25.Fi; 52.65.Kj; 52.20.Fs; 05.20.Dd

\section{Introduction}

Instabilities are ubiquitous in strongly ionized plasmas. They dominate the behavior of such systems and their study forms the core of the subject. The origin of the instabilities lies in the nature of the plasma interactions: on the one hand they are long range and thus can produce strong cooperative effects and on the other hand they become 'weaker' locally at high energies (or temperatures) as manifested by the decrease of the Coulomb cross section with energy rise ${ }^{1}$. The situation is different in weakly ionized cold plasmas, systems which have attracted much attention recently ${ }^{2,3}$. In such systems collective phenomena play a smaller role and instabilities are less common. Nevertheless there are cooperative phenomena in these systems too, which, as we have shown earlier ${ }^{4,5}$ for homogeneous idealized systems and will show here for more realistic laboratory situations, can lead to 
dramatic abrupt changes in the state of the plasma, when such systems are driven by external fields.

There is a large literature on the behavior of plasma in a cylindrical geometry (see for example Refs.6,7,8,9). The reason for considering this system again here is that we are interested in a regime in which the electrons are colder than in the self-sustained discharge plasmas generally investigated. The plasma has a sufficiently high degree of ionization to make the electron- electron and electron-ion collisions important. The condition of low electron temperature permits us to neglect inelastic collisions but requires that we include volume recombination effects.

The phenomenon in which we are most interested is a rapid, essentially abrupt, change in the electron temperature and current as the external electric field crosses a certain critical value which depends on the neutral-ion temperature, degree of ionization, etc. The phenomenon is related to the well known runaway effect in fully ionized plasmas caused by the decrease of the electron-ion collision cross section as the external field increases the electron energy ${ }^{10,11,12}$. In the partially ionized gas the presence of the neutrals prevents such a runaway. In fact, if one neglects collisions between the electrons (e-e), as is done in the swarm approximation ${ }^{13}$, valid when the degree of ionization is sufficiently small, then the stationary distribution (in the absence of inelastic collisions) will be of the Druyvesteyn form $^{14}$ for which the current and electron mean energy are smooth functions of the external field. The situation is similar when the size of the plasma is smaller than the energy relaxation length of the electrons ${ }^{9}$. There are, however, other regimes, even in weakly ionized plasmas, where the effect of the e-e collisions is sufficient to keep the electron distribution close to a Maxwellian ${ }^{10,4}$. Such conditions can lead to cooperative abrupt changes in the temperature and drift velocity of electrons as the external field is varied. The origin of the phenomenon is made clearer if one starts with a kinetic approach rather than with a macroscopic description.

While it is possible, even likely, that effects related to those discussed here have already been observed indirectly in the behavior of discharges it would be useful for both theoretical and practical reasons to have experiments in which the parameters can be controlled, so as to study the phenomena in a quantitative way. We expect that the transition will be seen as hysteresis in the current--voltage (I-V) characteristic of the plasma when the external electric field is slowly varying in time ${ }^{4,5}$.

We shall consider an experimental arrangement consisting of a weakly ionized gas in a tube of radius $R$ subjected to a constant external axial electric field $\mathbf{E}$. The plasma is assumed for simplicity to be produced through uniform ionization inside the tube, by some external source, at a constant rate $a$. It is balanced by two kinds of recombination processes: a bulk one and a surface process on the tube wall. The main bulk recombination for the regime we are interested in, are ${ }^{10}$ three body processes involving two electrons plus an ion, and dissociative recombinations in which a metastable atom-ion complex recombines with an electron. The rates depend on the temperatures and densities of the electrons, ions and neutrals in a rather complicated way; see Ref.15. For the sake of simplicity we lump the two processes together and assume an effective rate of bulk recombination proportional to $T^{-3 / 2}$, where $T$ is the electron temperature. We ignore the dependence of this rate on the neutral and ion temperature and on the pressure 
which we keep more or less constant. The recombination at the wall is also treated phenomenologically. In particular we assume that the energy is absorbed by the wall which is kept at a fixed temperature, see section 4 .

We have in mind here a situation in which the great majority of neutral atoms are some kind of noble gas to which may, or may not, be added a small amount of a more easily ionized second species, though we realize that in the latter case the analysis would be more complicated $^{16}$. This will be reflected mainly in the rates of ionization $a$ and recombination $\gamma$ since we shall always consider a regime in which the density of electrons $n(r, t)$ is much lower than that of the neutrals, $N$, but big enough, due to the great disparity between the electron mass, $m$, and the ion-neutral mass, $M$, for binary electron-electron collisions to dominate the energy exchanges in electron-ion and electron-neutral (e-i and e-n) collisions. This requires ${ }^{4}$ that

$$
\frac{\sigma m}{2 \pi M e^{4}}(k T)^{2}<<\frac{n}{N}<<1
$$

Here $k$ is the Boltzmann constant, and $\sigma$ is the total electron-neutral particle collision cross section, which is taken to be a constant in our work. Putting in appropriate values for the parameters on the left side of (1a) gives (see Ref.4),

$$
1>>n / N>>8 \cdot 10^{-7}, 4 \cdot 10^{-8}, 1.5 \cdot 10^{-8}
$$

for $\mathrm{He}, \mathrm{Ne}$, Ar plasmas respectively, when $k T$ is approximately $1 \mathrm{eV}$ and it decreases as $T^{2}$ for colder electrons. The upper bound relates to the fact that we ignore any collective self induced electrostatic or magnetic interactions.

The ions in our model are assumed to have a uniform temperature, $T_{i}$, the same as the neutrals, while their density is $n(r, t)$, i.e. the plasma is treated as locally quasi-neutral. We assume axial symmetry and longitudinal homogeneity so the spatial dependence is only in the radial variable $r \leq R$. The different mobilities of ions and electrons are compensated by an internally generated radial ambipolar electric field $\mathbf{F}(r, t)$. We are thinking of a quiescent, long positive column which fills the tube ${ }^{6}$.

The reason for considering external rather than field induced ionization is that in our previous works ${ }^{4,5}$, in which we considered a spatially homogeneous case with an a priori fixed plasma density, we found that as we varied the external field $E$, there was a transition in the electron distribution between regimes of weak and strong coupling to the ions. (Ref.5 presents a rigorous proof of such a transition for a greatly simplified model system). This mechanism of a kinetic transition, which we investigate here in a more realistic physical situation, requires a low electron temperature and relatively weak electric field, which seems hard to achieve when the ionization is produced by the field, see Sec.4.

The basic idea in Ref.4 and here is to consider situations in which the collisions between electrons are strong enough to force their velocity distribution $f(r, \mathbf{v}, t)$ to stay close to a Maxwellian $M_{f}$ with temperature $T$ and drift velocity $\mathbf{w}$. The values of $T$ and w, which are simply related to the first two velocity moments of $f$, are then determined by self consistent "hydrodynamic" equations, i.e. we evaluate the integrals entering the time evolution of $T$ and $\mathbf{w}$ with the help of replacing $f$ by this Maxwellian. For the spatially 
homogeneous case this yields ordinary differential equations in time for $T$ and $\mathbf{w}$ which can be reduced to a couple of transcendental equations for stationary values $T(E)$ and $\mathbf{w}(E)$ yielding, in some cases, S-shaped curves as functions of $E=|\mathbf{E}|$. In the region where $w$ decreases with $E$ the system is unstable. The current and the electron temperature can therefore be expected to jump upwards (downwards) when the field increases (decreases). This will occur at different values of the field intensity, see Fig.3: in the lower (upper) part of the loop the electrons will be supercooled (superheated) creating in this way a hysteresis loop. The origin of this behavior lies in a changeover from e-i to e-n coupling as the dominant factor when the electron energy is increased by the field.

In the situation analyzed in this paper we obtain nonlinear partial differential equations for $n(r, t), T(r, t), \mathbf{w}(r, t)$ and $F(r, t)$. Their stationary solutions are the main concern of this work. This is presented in section 3 following the mathematical formulation of the problem in section 2. A discussion of physical situations where the transition predicted by the two-fluid model used in this paper might be observed is presented in section 4. This is a much more restrictive domain than that given by (1), it requires essentially $n / N \sim 10^{-4}$ while $N \sim 10^{15} \mathrm{~cm}^{-3}$ for light noble gases when $T_{i}$ is close to room temperature. At the transition the electron temperature jumps within a range of several $T_{i}$, staying significantly lower than the usual temperatures in self-sustaining gas discharges. This determines the experimental set up we study here. The nature of the various approximations made is discussed in section 4 and in the Appendix.

\section{Mathematical Description}

The behavior of the system is described by kinetic equations for the electron and ion distribution functions $f(r, \mathbf{v}, t)$ and $f_{i}(r, \mathbf{v}, t)$ normalized to the same density $n(r, t)$. Under the above assumptions the Boltzmann equation for electrons has the form ${ }^{4}$

$$
\begin{gathered}
\frac{\partial f(r, \mathbf{v}, t)}{\partial t}-\frac{e}{m}\left[\mathbf{E}+\frac{\mathbf{r}}{r} F(r, t)\right] \cdot \nabla_{\mathbf{v}} f(r, \mathbf{v}, t)+\mathbf{v} \cdot \nabla_{\mathbf{r}} f(r, \mathbf{v}, t)= \\
a \psi(\mathbf{v})-\gamma n f(r, \mathbf{v}, t)+\frac{m}{M v^{2}} \frac{\partial}{\partial v}\left\{\left(b n+v^{4} / l\right)\left[\bar{f}(r, v, t)+\frac{k T_{i}}{m v} \frac{\partial \bar{f}(r, v, t)}{\partial v}\right]\right\}+ \\
\frac{b n}{v^{3}} \hat{L} f(r, \mathbf{v}, t)+\frac{v}{l}[\bar{f}(r, v, t)-f(r, \mathbf{v}, t)]+Q[f] .
\end{gathered}
$$

In (2) the first two terms on the right side represent the ionization and recombination respectively: $\psi(\mathbf{v})$ is the normalized distribution function of newly born electrons with mean kinetic energy $m v_{0}^{2} / 2$,

$$
\int \psi(\mathbf{v}) d^{3} v=1, \quad \int \mathbf{v} \psi(\mathbf{v}) d^{3} v=0, \quad \int v^{2} \psi(\mathbf{v}) d^{3} v=v_{0}^{2} .
$$

The third term with the prefactor $m / M$, represents the diffusion in the speed of the electrons due to collisions with ions and neutrals. The effectiveness of the e-i collisions is

proportional to $n$ and is strongly peaked at small speeds. The constant $b$ is given ${ }^{4,11}$ by $b=4 \pi e^{4} L / m^{2}(L \sim 10$ is the Coulomb logarithm), while $l=1 / N \sigma$ is the mean free path 
of electrons in e-n collisions. The next two terms in the right hand side of (3) represent respectively the effects of e-i and e-n collisions on the angular parts of electron velocities with

$$
\hat{L}=\Sigma_{\mu, \nu} \frac{\partial}{\partial c_{\mu}}\left(c^{2} \delta_{\mu, \nu}-c_{\mu} c_{\nu}\right) \frac{\partial}{\partial c_{\nu}}, \quad(\mu, \nu=1,2,3)
$$

where $\mathbf{c}=\mathbf{v}-\mathbf{W}$ and $\mathbf{W}$ is the ion drift velocity. We neglect the difference between $|\mathbf{c}|$ and $|\mathbf{v}|$, in particular in the energy exchange term in (2), since $|\mathbf{W}|<<v$. For the e-n collisions we have defined

$$
\bar{f}(r, v, t)=\frac{1}{4 \pi} \int f(r, \mathbf{v}, t) d \Omega_{v}
$$

as the sphericalized average of $f(r, \mathbf{v}, t)$.

For simplicity we have written the e-i and e-n collision terms when ions and neutrals have approximately the same mass $M$. In the case of using an easily ionized impurity these collision terms have to be modified in an obvious way. However, as long as the density of these impurities is of order of $n$, and $n / N<<1$, we do not expect them to substantially affect our result. Finally the last term $Q[f]$ represents e-e collisions. We do not specify here the exact form of this term (although we have in mind a Landau form ${ }^{10,11,12}$ ), since it conserves the hydrodynamical moments corresponding to density, mean velocity and kinetic energy which we are going to derive from (3).

We could but do not write down a similar kinetic equation for the ions. As already mentioned we assume them to have a distribution close to a Maxwellian with a uniform temperature $T_{i}$, equal to that of the neutrals. The assumption of charge neutrality further implies that both electrons and ions have the same density

$$
n(r, t)=\int f(r, \mathbf{v}, t) d^{3} v=\int f_{i}\left(r, \mathbf{v}_{\mathbf{i}}, t\right) d^{3} v_{i}
$$

and the same radial drift velocity, as we shall see in the next section. The maintenance of this quasi-neutrality is the task of the radial ambipolar field $F(r)$. The effect of recombination at the wall and other wall effects will be discussed in the next section.

\section{Hydrodynamic Description}

Many properties of the plasma can be expressed in terms of the first few velocity moments of $f$ and $f_{i}$. To obtain these we multiply (2) by $1, \mathbf{v}, v^{2}$ and integrate over $\mathbf{v}$. This yields expressions for the time derivatives of the density $n(r, t)$, drift velocity $\langle\mathbf{v}\rangle=\mathbf{w}(r, t)$ and $\left\langle v^{2}\right\rangle=3 k T(r, t) / m+w^{2}(r, t)$ which however involve unknown integrals over $f$. To obtain a closed set of hydrodynamical equations we follow the procedure in Refs.4,5, which is often used for collisional plasmas, and compute all integrals over $\mathbf{v}$ with the help of the replacement

$$
f(r, \mathbf{v}, t) \rightarrow M_{f}(r, \mathbf{v}, t)
$$

where

$$
M_{f}(r, \mathbf{v}, t)=n(r, t)(m / 2 \pi k T)^{3 / 2} \exp \left[-m(\mathbf{v}-\mathbf{w})^{2} / 2 k T\right]
$$


is a local Maxwellian with the same $r$ and $t$ dependent first moments, $n, \mathbf{w},\left\langle v^{2}\right\rangle$, as $f(r, \mathbf{v}, t)$. This assumption is motivated by the fact that the local Maxwellian $M_{f}$ is the unique distribution which makes the e-e collision term $Q[f]=0$ and should thus be a good approximation (after some transient time) for situations in which $Q[f]$ is the dominant term in determining the shape of the distribution function. This requires, at the minimum, that $n / N$ satisfy (1b) and that $E, a$ and $\gamma$ be small so that the energy exchanges in the e-e collisions are most important in determining the shape of the energy distribution of the electrons; the effectiveness of the e-n and e-i collisions being reduced by the factor $m / M$ which is the physically small parameter in the problem. Eq. (5) can be rigorously justified, as was shown in Ref.5, for sufficiently idealized models. We believe that the evaluation of these integrals via $M_{f}$ remains valid, at least approximately, in certain realistic situations, see section 4 and Appendix.

Using (4) we obtain four equations for the electron density $n$, the longitudinal and radial components of $\mathbf{w}, w_{z}, w_{r}$, the temperature $T$, and the radially directed electric field F. In writing down these equations we shall neglect higher order terms in the drift velocity $w(r)$ as compared to the electron thermal velocity $u(r)=\sqrt{3 k T / m}$. (This is a good approximation when $m<<M$, see section 4 and Appendix).

a) Electron density equation

$$
\frac{\partial n}{\partial t}=-\frac{\partial}{r \partial r}\left(r n w_{r}\right)+a-c \frac{n^{2}}{u^{3}}
$$

b) Electron flux in the z-direction

$$
\frac{1}{n} \frac{\partial\left(n w_{z}\right)}{\partial t}+\frac{e E}{m}=-2 \sqrt{\frac{6}{\pi}} w_{z} \frac{b n+4 u^{4} / 9 l}{u^{3}}-\frac{c n w_{z}}{u^{3}} .
$$

c) Electron flux in the radial direction

$$
\frac{1}{n} \frac{\partial\left(n w_{r}\right)}{\partial t}+\frac{e F(r, t)}{m}=-\frac{8}{9} \sqrt{\frac{6}{\pi}} w_{r} \frac{u}{l}-\frac{c n w_{r}}{u^{3}}-\frac{1}{3 n} \frac{\partial}{\partial r}\left(n u^{2}\right) .
$$

d) The energy balance equation

$$
\begin{gathered}
\frac{1}{2} \frac{\partial\left(n u^{2}\right)}{\partial t}+n \frac{e}{m}\left[F(r, t) w_{r}+E w_{z}\right]+\frac{5}{6 r} \frac{\partial}{\partial r}\left(r n w_{r} u^{2}\right)= \\
\frac{a v_{0}^{2}}{2}-\frac{c n^{2}}{2 u}-\sqrt{\frac{6}{\pi}} \frac{m}{M}\left(1-\frac{v_{i}^{2}}{u^{2}}\right) n \frac{b n+8 u^{4} / 9 l}{u}
\end{gathered}
$$

Here

$$
u^{2}(r, t)=3 k T(r, t) / m=\left\langle v^{2}\right\rangle-w^{2}(r), \quad v_{i}^{2}=3 k T_{i} / m,
$$

and we have set $\gamma$ proportional to $T^{-3 / 2}, \gamma=c / u^{3}$, see Ref.6. The temperature independent parameter $c$ describes qualitatively the rate of the volume recombination. 
In the case of dissociative recombination $c$ is typically ${ }^{10,15}$ in the range $4 \times\left(10^{13}-\right.$ $\left.10^{15}\right) \mathrm{cm}^{6} / \mathrm{sec}^{4}$ and close to the lower value for pure Helium. The temperature dependence of $\gamma$ represents a combination of both dissociative process and three-body recombinations. The latter will be most important when the electron density $n$ is large and $T$ is low. It gives a reasonable rate in the vicinity of $300^{\circ} \mathrm{K}$ for the neutral and ion temperatures.

A similar procedure with the ion kinetic equation yields (6) for the zeroth moment because charge neutrality implies the same $n(r, t)$ and therefore the same $w_{r}(r, t)$. The ion radial flux is described by the following equation

$$
\frac{1}{n} \frac{\partial\left(n w_{r}\right)}{\partial t}+\frac{e F(r, t)}{M}=-\frac{8}{9} \sqrt{\frac{6}{\pi}} w_{r} \frac{U}{l_{i}}-\frac{c n w_{r}}{u^{3}}-\frac{1}{3 n} \frac{\partial}{\partial r}\left(n U^{2}\right)-\frac{1}{n r} \frac{\partial}{\partial r}\left(r n w_{r}^{2}\right)
$$

Here $U$ is the ion thermal velocity $\left(M U^{2}=3 k T_{i}\right)$ and $l_{i}$ is the mean free path in i-n collisions with respect to momentum relaxation. Unlike in (8) we cannot, in (10), neglect $w_{r}^{2}$ compared with $U^{2}$. We do however neglect the ionic contribution to the total longitudinal current and assume that the ions have the same temperature as the neutrals since they are in a much better thermal contact with the neutral particles than the electrons. We believe that in our range of electron temperatures we make only a small error in taking $T_{i}=$ const and neglecting the ion heating. Dependence of $T_{i}$ on $r$ can be easily incorporated by adding one more differential equation similar to (9).

The nonlinear partial differential Eqs.(6)-(10) have to be solved for the five unknown functions $F(r), n(r), u(r), w_{z}(r), w_{r}(r)$. In the stationary state the set $(6-10)$ can be reduced by the elimination of $w_{z}(r)$ and $F(r)$ with the help of (7),(8) and (10). We obtain

$$
w_{z}(r)=-\frac{1}{2} \sqrt{\frac{\pi}{6}} \frac{e E}{m} \frac{u^{3}(r)}{g n(r)+4 u^{4}(r) / 9 l}
$$

and three coupled equations

$$
\begin{gathered}
\frac{d}{r d r}\left(r n w_{r}\right)=a-c \frac{n^{2}}{u^{3}} \\
\frac{m}{M} \frac{d}{d r}\left(n \frac{u^{2}+v_{i}^{2}}{3}\right)+\frac{d}{r d r}\left(r n w_{r}^{2}\right)=-\left(\frac{8}{9} \sqrt{\frac{6 m}{\pi M}} \frac{v_{i}}{l_{i}}+\frac{c n}{u^{3}}\right) n w_{r}, \\
u(r) n(r) \frac{d u(r)}{d r}-\frac{1}{3} u^{2}(r) \frac{d n(r)}{d r}=\frac{8}{9 l} \sqrt{\frac{6}{\pi}} n w_{r}+A(r) / w_{r}
\end{gathered}
$$

for $n(r), u(r), w_{r}(r)$. Here $g=b+\frac{1}{2} \sqrt{\frac{\pi}{6}} c$ and

$$
\begin{gathered}
A(r)=\frac{1}{2} \sqrt{\frac{\pi}{6}}\left(\frac{e E}{m}\right)^{2} \frac{u^{3}(r) n(r)}{g n(r)+4 u^{4}(r) / 9 l}+\frac{a}{2}\left[v_{0}^{2}-\frac{5}{3} u^{2}(r)\right]+\frac{1}{3} c \frac{n^{2}(r)}{u(r)}- \\
\sqrt{\frac{6}{\pi}} \frac{m}{M}\left[1-\frac{v_{i}^{2}}{u^{2}(r)}\right] n(r) \frac{b n(r)+8 u^{4}(r) / 9 l}{u(r)} .
\end{gathered}
$$


It is well known ${ }^{6,7,8}$ that in the hydrodynamic approximation the assumption of the charge neutrality leads to singularities near the plasma boundaries due to the space charge in the plasma sheath caused by the different thermal velocities of electron and ions and e-i recombinations near the wall. The singularity manifests itself in Eqs.(12-14) through the determinant of the linear system for the derivatives $\frac{d n}{d r}, \frac{d u}{d r}, \frac{d w_{r}}{d r}$ becoming zero when the radial drift velocity, at some $r=r^{\prime}$, reaches the value

$$
w_{r}=\sqrt{k \frac{5 T_{e}+3 T_{i}}{3 M}}
$$

that is close to the adiabatic sound speed ${ }^{7}$. According to (8) the infinite derivatives imply an infinite electric field $F(r)$ and hence a breakdown of the model. Following Refs.7,8 we interpret this to mean that charge neutrality cannot hold for $r>r^{\prime}$ and $r^{\prime}$ marks the boundary of the plasma sheath. On the other hand the thickness of the sheath is ${ }^{6}$ of the order of a few Debye lengths $\lambda_{D}=\sqrt{k T_{i} / 4 \pi n e^{2}} \approx 10^{-4} \mathrm{~cm}$ when $T_{i}=300 \mathrm{~K}, n \approx 10^{12} \mathrm{~cm}^{-3}$. Assuming that $R>>\lambda_{D}$ we can neglect the difference between $r^{\prime}$ and $R$ in Eq.(16) and instead use (16) as the boundary condition for (12-14), as is proposed by Persson ${ }^{7}$ and other authors (see Refs.6,8).

Our task now is to solve (12)-(14) subject to the conditions

$$
\frac{d n}{d r}(0)=0, \quad \frac{d u}{d r}(0)=0, \quad w_{r}(0)=0
$$

at $r=0$ and to $w_{r}(R)$ given by (16) at $r=R$. The functions $n(r), u(r)$ are finite on the tube axis, $r=0$, Eqs.(12), (14), (17) therefore imply

$$
\frac{d w_{r}}{d r}(0)=\frac{1}{2 n(0)}\left[a-c n^{2}(0) / u^{3}(0)\right]
$$

and $A(0)=0$. The last relationship is very important and we rewrite it as

$$
\begin{gathered}
\sqrt{\frac{\pi}{6}}\left(\frac{e E}{m}\right)^{2}=\frac{g n(0)+4 u^{4}(0) / 9 l}{u^{3}(0) n(0)}\left\{2 \sqrt{\frac{6}{\pi}} \frac{m}{M}\left[1-\frac{v_{i}^{2}}{u^{2}(0)}\right] n(0) \frac{b n(0)+8 u^{4}(0) / 9 l}{u(0)}\right. \\
\left.-a\left[v_{0}^{2}-\frac{5}{3} u^{2}(0)\right]-\frac{2}{3} c \frac{n^{2}(0)}{u(0)}\right\} .
\end{gathered}
$$

Solving the set (12-14) with conditions (16-19) we determine the density and temperature profiles as well as the average temperature $\bar{T}(E)$ and the total longitudinal current $I(E)$ :

$$
\bar{T}(E)=\frac{2 m}{3 k R^{2}} \int_{0}^{R} u^{2}(r) r d r, \quad I(E)=2 \pi e \int_{0}^{R} r n(r) w_{z}(r) d r .
$$

When we studied in Ref.4 the spatially homogeneous problem without ionization and recombination, we had $c=0, a=0, n=$ const, $u=$ const, and (19) served as the equation 
for the determination of $u$, or the electron temperature, as a function of $E$. We have a similar situation here when $l / R<<1$. In this case one can disregard the recombination on the tube walls and we have again a spatially homogeneous problem $n=n(0), u=u(0)$. The only difference is that we have now, in virtue of (12),

$$
n=\sqrt{\frac{a u^{3}}{c}}
$$

Defining

$$
\phi=\frac{9}{8} \sqrt{\frac{\pi}{6}} \frac{l v_{i}^{-3 / 2}}{\sqrt{a c}}\left(\frac{e E}{m}\right)^{2}, \omega=\frac{9}{4} b l v_{i}^{-5 / 2} \sqrt{\frac{a}{c}}, x=\frac{u}{v_{i}}, \theta=\frac{v_{0}^{2}}{v_{i}^{2}}, \mu=\frac{c}{b}, \epsilon^{2}=\frac{m}{M}
$$

(19) can be rewritten in the dimensionless form

$$
\phi=\frac{\omega+x^{5 / 2}}{x^{3}}\left[\sqrt{\frac{6}{\pi}} \frac{\epsilon^{2}}{\omega \mu}\left(x^{2}-1\right)\left(\omega+2 x^{5 / 2}\right)+\left(x^{2}-\Theta\right) / 2\right]
$$

It is obvious from $(22)$ that $\phi$ is not monotone in $x$ and therefore $T(E)$ is not singlevalued if the parameter $\omega$ is big enough. In a Helium plasma where $M / m \approx 7000$, we take $c=4 \cdot 10^{13} \mathrm{~cm}^{6} \mathrm{sec}^{-4}, \theta=10$ and find that $\omega$ should be larger than about 113 in order to have a transition corresponding to an S-shaped $T(E)$ curve; see Fig.1, where $\omega=250$. For smaller $c$ the critical value of $\omega$ is lower.

In terms of the ion temperature and the degree of ionization $\omega$ can be represented as

$$
\omega=\pi L \frac{e^{4}}{\sigma\left(k T_{i}\right)^{4}} \frac{n}{N}
$$

This is equal approximately $2 \cdot 10^{6} n / N$ for Argon with the ambient temperature of the background gas. We see that the transition requires $n / N \sim 10^{-4}$ or higher. Real values for the electric field and current density $j$ can be found with the help of following relations:

$$
E l=1.28 \cdot 10^{-4}\left(\frac{T_{i}}{300}\right) \sqrt{\omega \phi}, \quad j l=2.6 \cdot 10^{-3}\left(\frac{T_{i}}{300}\right)^{5 / 2} \frac{x^{3} \sqrt{\omega^{3} \phi}}{\omega+x^{5 / 2}} .
$$

Here $l$ is in $\mathrm{cm}, E$ and $j$ are in volts $/ \mathrm{cm}$ and $\mathrm{mA} / \mathrm{cm}^{2}$ respectively.

\section{The $\mathbf{T}=$ const Approximation}

As a first step in solving the nonuniform case (12-14) let us assume that the electron temperature is constant, $u(r)=u(0)$. It seems reasonable to study such a simplified problem both as a guide for the more general case which we shall consider later and as an approximation which often yields reliable results for the positive plasma column ${ }^{6}$.

The energy balance equation (14) cannot hold now for all $r \leq R$, but at the point $r=0$ it reduces to $(19)$, and our task is to solve $\mathrm{n}(12,13)$ with the conditions $(16-18)$. For 
fixed $\mathrm{E}$ and $n=n(0) \mathrm{Eq} .(19)$ determines $u$ and therefore the electron temperature in the tube. We then integrate $(12,13)$, find the profiles $n(r), w_{r}(r)$, compute $w_{z}(r)$ with the help of (11), and using (20) find the total current $I(E)$, which gives the current-voltage characteristic of the plasma. We can neglect the difference between $b$ and $g$, because in noble gases $g / b-1 \sim \mu \sim 10^{-4}$.

To solve Eq. $(12,13)$ numerically we pick a value of $u$ and find a suitable $n(0)$ which allows to satisfy (16). Eq. (19) is used for the calculation of $E$ for each choice of $u$ and $n(0)$. In this way we obtain the functions $n(r), w_{z}(r)$ and substitute them into (20). Fig.2 shows the electron temperature and the electric current thus computed versus $E^{2}$ in relative units when $l / R=0.2$. Curves $T(E)$ are single-valued for $\omega \leq 66$ with the same $\Theta, c$ as before in a Helium plasma.

\section{$\mathbf{T} \not \equiv$ const}

We solved numerically Eqs. (12)-(14) with boundary conditions (16-19). Technically we choose for each $u(0)$ a trial value of $n(0)$, compute $\mathrm{E}$ with the help of Eq.(19), and solve the differential equations $(12-14)$ for this triple. The resulting $w_{r}(r)$ will generally not satisfy the boundary condition (16) at $r=R$ and we then iterate with a different $n(0)$. This search can be easily optimized, the procedure usually converges very fast and yields the profiles of $n(r)$ and $u(r)$ for $r \leq R$. From these we can obtain $F(r), w_{z}(r), w_{r}(r)$ for a given external field $\mathrm{E}$. In addition we also find the total current and the mean electron temperature over the tube cross section (20). The parts of the $T(E)$ and $I(E)$ curves with negative derivatives are unstable and physically non-accessible ${ }^{4,5}$, which also shows up in the computation being extremely unstable there when we try to find the solutions numerically. Thus for $E=E^{\prime}$ in Fig.3 and any trial temperature on the tube axis inside the gap region $(0.046-0.065 \mathrm{~V}), T(r)$ immediately jumps to a point corresponding to $T^{\prime}$ after a few steps of integration. This difficulty does not occur when we keep $T=$ const.

A qualitative description of the results is as follows:

a) $n(r)$ always decreases monotonically. For some range of parameters a kinetic transition takes place, $n(0)$ is then not a single-valued function of the external field or the mean electron temperature. The decrease of the volume recombination rate $c$ enhances the transition.

b) The I-V characteristic for the total current $\mathrm{I}(\mathrm{E})$ and the mean electron temperature show transition like behavior for $\omega>60$. This is close to that obtained for the model $T=$ const

c) $T(r)$ is a smooth monotonically decreasing curve when $E$ is large; for very small $E$ the electron temperature passes a maximum at some $r<R$. This maximum is caused by the electron heating in the ambipolar radial field $F$ which is equal to zero on the tube axis. At stronger fields $E$ this effect is disguised by the external field producing more heat near the axis where the electron density is higher.

d) The radial drift of electrons and ions as well as the ambipolar electric field $F(r)$ rise strongly near the tube walls, the latter even goes to infinity in this model but the wall potential with respect to the tube axis is finite as one can see from Eq.(8). 
We present a few curves for $\omega=90$ in Figs.3,4 which illustrate the transition, the temperature profiles across the tube cross section and the radial dependence of the transverse electric field $F(r)$. As $E$ is varied we expect jumps in $T(E)$ and $I(E)$ at the arrows in Fig.3.

\section{Discussion}

In this work we investigated properties of the stationary state of a partially ionized plasma in a cylindrical tube maintained by a source of ionization and subjected to an external axial electric field $E$. Our calculations were done in a collisional two-fluid scheme. The neutrals are treated as a given background with fixed density $N$ and temperature $T_{i}$ while the ions were assumed to have the same uniform temperature $T_{i}$ and a density $n(r)$ equal to that of the electrons, i.e. charge neutrality in the interior of the tube.

The assumption that the heating of the electrons by the external and ambipolar fields together with the injection of relatively warm electrons in the process of ionization does not heat the neutrals and ions very much seems reasonable when one takes into account the density and heat conductivity of the neutrals and the strong coupling between them and the ions. To see this in a semiquantitative way we note that the temperature distribution for the neutrals is governed by the equation

$$
\frac{1}{r} \frac{d}{d r}\left(r \frac{d T}{d r}\right)=-\frac{P(r)}{\lambda}
$$

where $P(r)$ describes the heating sources and $\lambda$ is the thermal conductivity which can be taken to be constant. By keeping the walls of tube at a given temperature $T_{0}$ we impose the boundary condition $T(R)=T_{0}$ which gives

$$
T(r)=T_{0}+\frac{1}{\lambda} \int_{r}^{R} \frac{d x}{x} \int_{0}^{x} y P(y) d y
$$

Let us consider three cases: 1) $P(r)$ is a constant, 2) linearly falls to zero, and 3) approaches zero parabolically. Then letting

$$
\bar{P}=\frac{2}{R^{2}} \int_{0}^{R} P(r) r d r
$$

be the average intensity of the heat source, Eq.(24) yields, on the tube axis

$$
T_{1}(0)=T_{0}+\frac{1}{4} \frac{\bar{P} R^{2}}{\lambda}, T_{2}(0)=T_{0}+\frac{5}{12} \frac{\bar{P} R^{2}}{\lambda}, T_{3}(0)=T_{0}+\frac{3}{8} \frac{\bar{P} R^{2}}{\lambda}
$$

respectively. The condition

$$
\bar{P}<<2 \lambda T_{0} / R^{2}
$$

then guarantees an approximately uniform temperature of the neutrals. In fact we take $T_{0}=T_{i}$ and Eq.(25) can be rewritten in the form

$$
E \bar{j}<<\frac{1}{\sigma R^{2}} \sqrt{\frac{3}{M}}\left(k T_{i}\right)^{3 / 2}
$$


if, for simplicity, we neglect the effects of the neutral gas non-ideality and only take into account the heating by the external field, $P=E j$. For a typical example of the situation considered in this paper, which will be described later in this section, $E \bar{j}$ is four orders of magnitude smaller than the right hand side of (25).

The assumption of a constant ion temperature will hold only approximately. We expect that away from the tube axis the ion temperature will become somewhat larger than the temperature of neutrals but not significantly so, except maybe in close vicinity of the wall ${ }^{6,7,8}$. Since the transition we are studying here takes place when $T$ is only a few times higher than that of the neutrals, we may assume a constant ion temperature $T_{i}$, at least for the majority of ions.

The kinetic model on which the hydrodynamic equations (6-10) are based is the nonlinear Boltzmann equation (2) for the electron distribution function $f(r, \mathbf{v}, t)$. To obtain the fluid equations we have used the approximation of replacing $f$ by $M_{f}$ in evaluating the integrals for the time derivatives of the hydrodynamic variables. The justification given in section 3 is the dominant effect of e-e collisions. But, as is clear from the behavior of the e-e collision cross section, which decreases for large speeds as $v^{-4}, f(v)$ will not be uniformly close to $M_{f}(v)$ for all $v$. For large speeds, the behavior of $f$ in the stationary state will be dominated, for $|E|$ not very small, by collisions with neutrals which lead to a Dryuvesteyn type tail of the form ${ }^{14} f \sim e^{-A v^{4} / E^{2}}$. Very slow electrons, on the other hand, are strongly influenced by the e-i interactions and their distribution is distorted near $v=0$ also. However, since we are only interested in the evaluation of averages of quantities which are not too peaked at very slow or very high speeds, the main contribution will come from typical values of $\mathbf{v}, v \sim u=\sqrt{3 k T / m}$, where the bulk of electrons are. In this region of velocity space $f$ will be close to $M_{f}$ whenever the "relaxation time" $\tau_{e e}$ associated with e-e collisions is small compared with other relaxation times in the problem which would try to drive the electron distribution away from a local Maxwellian. We now evaluate the different relaxation times for typical electrons in the spirit of plasma kinetic theory ${ }^{11,12}$.

The frequency of e-e collisions ${ }^{1}$ is $b n / u^{3}$ so $\tau_{e e}$, the time of energy and momentum relaxation in such collisions, can be approximated as

$$
\tau_{e e} \approx \frac{u^{3}}{b n} .
$$

The relaxation time of the newly created electrons, whose mean speed $v_{0}$ is higher than $u$, can be obtained from (26) by replacing $u$ with $v_{0}=\Theta^{1 / 2} u$. On the other hand an electron with speed $v$ loses, in a collision with a slow ion having velocity $u_{i}$, a fraction $2 m / M$ of its energy. Such collisions take place with frequency, $\nu_{e i}=b n / u^{3}=\tau_{e e}{ }^{-1}$, so the e-i energy relaxation time is

$$
\tau_{e i} \sim \frac{M}{m} \tau_{e e} .
$$

By the same reasoning, the energy relaxation time with neutrals, where the collision frequency $\nu_{e n}$ is $v / l$, is

$$
\tau_{e n} \approx M / m \nu_{e n}=M l / m u .
$$

We thus always have $\tau_{e e} / \tau_{e i}=m / M<<1$ and the requirement that $\tau_{e e} / \tau_{e n}<<1$ is just the left side inequality in (1a). This assures that e-e collisions will play a dominant role 
in determining the energy distribution of the electrons, at least for the case of a uniform plasma with fixed electron density $n$, considered in Refs.3-5. In the present case we also have to consider the electron lifetimes relative to recombination in the bulk, $\tau_{B}$, and on the wall $\tau_{W}$. A simple calculation yields

$$
\tau_{B}=\frac{u^{3}}{c n}=\frac{b}{c} \tau_{e e} \approx 10^{4} \tau_{e e} .
$$

To estimate $\tau_{W}$ we compare the flux of neutralizing ions to the walls, $u_{i} n(R) \cdot 2 \pi R$, and the total number of electrons in the tube cross section $\bar{n} \pi R^{2}: \tau_{W} \approx R \bar{n} / 2 u_{i} n(R)$. Taking roughly $n(R) / \bar{n} \sim 1 / 20$ we obtain, after some substitutions,

$$
\tau_{W} \approx 10 R / u_{i} \approx 10(R / l)\left(M T / m T_{i}\right)^{\frac{1}{2}} \tau_{e n} .
$$

Thus for $R / l \geq 100, \tau_{W} \geq \tau_{e n}$ and the arguments used for fixed $n$ should apply.

We note here however that in order to get $M \sim M_{f}$ with both $T$ and $\mathbf{w}$ determined by the hydrodynamic equations, one actually needs to assume also that $\tau_{e e}$ is small compared to the momentum relaxation times, i.e. that $\left(\tau_{e e} \nu_{e i}\right)$ and $\left(\tau_{e e} \nu_{e n}\right)$ are small. This was assumed for the simplified model equations ${ }^{5}$ but clearly does not hold in the plasma. A better approximation scheme to solving (2) would therefore be to take $f \sim M_{f}^{0}$, a local Maxwellian with mean drift $\mathbf{w}=0$. The leading corrections to this Maxwellian would be of order $\sqrt{m / M}$, and would have to be computed from a linearization of (2) about $M_{f}^{0}$. The result would then be the input for the self-consistent evaluation of $T$. This is what was essentially done heuristically and approximately in Ref.4. To make the scheme work rigorously the effective strength of $E$ and hence $w$ must also be of this order. This is indeed what we find here, for the range of $E$ in Figs. 1-3, with $w / u \sim \sqrt{m / M}$. In fact the results obtained in Ref.4 are qualitatively similar to those obtained with the present approximation scheme for uniform systems, which facilitates computation; see also Appendix.

\section{Realizability of Transition}

We shall consider now, within the scheme of section 3 and the even simpler relaxation time estimates discussed above, the physical conditions necessary for observing the kinetic transition manifested in the $S$-shaped curves in Figs. 1-3. Since the origin of this behavior is a changeover from e-i to e-n collisions as the dominant mechanism for the dissipation of the energy which electrons gain from the external field, it is necessary that at the initiation of the transition, i.e. at the bottom part of the $S$-curve, the electron temperature, $T_{b}$, should be low enough for $\nu_{e i} / \nu_{e n}$ to be large. The requirement that this ratio, which can be written as $(4 \pi n L / 9 N)\left(e^{4} / \sigma\right) /\left(k T_{b}\right)^{2}$, be large compared to one and that we still have $n / N<<1$ imposes a strong constraint on the temperature of the electrons $T_{b}$. Assuming a Helium plasma with the neutrals and ions at room temperature, $T_{i}=300^{0} \mathrm{~K}$,

$R=1 \mathrm{~cm}, \theta=\left(v_{0}^{2} / v_{i}^{2}\right)=10$, corresponding to an energy of $1 / 4 \mathrm{eV}$ for newly created electrons, $\sigma=5 \times 10^{-16} \mathrm{~cm}^{2}$ (see Ref.17) the ionization fraction at the center of the tube, corresponding to the bottom in Fig. 3 is, $n / N \sim 4 \cdot 10^{-5}$. We took $l / R=0.2$ which implies $N \approx 10^{16} \mathrm{~cm}^{-3}$ corresponding to $N k T_{i}=10^{-1}$ Torr and $n(0) \sim 4 \cdot 10^{11} \mathrm{~cm}^{-3}$. The electric field in Fig.3 goes from zero to about $0.2 \mathrm{~V} / \mathrm{cm}$ and the total current does not 
exceed $4 m A$. For a Neon plasma $\sigma$ is smaller by a factor of 9 and so is the requirement on the ionization fraction.

Whether conditions like these can be achieved experimentally is at the moment an open question. What is clear however is that if we have to rely on ionization caused by the external field $E$ then $T_{b}$ will be about $1 \mathrm{eV}$ and the electrons will never be coupled strongly to the ions for $n / N<<1$. To have a chance of seeing a transition corresponding to a crossover from e-i to e-n coupling we would have to consider an almost fully ionized plasma in which the remaining neutrals would prevent the runaway effect caused by the external field $E$ when only e-e and e-i collisions are considered, (see Ref.11).

To gain a better understanding of the dynamics involved in this non-equilibrium kinetic transition, let us analyze, in simple physical terms, the stationary state of our system in the presence of the electric field $\mathbf{E}$. We assume as before that in each e-i or e- $\mathrm{n}$ collision the fraction of energy lost is $2 m / M$ and the direction of motion of the colliding electron is randomized. Under the action of the force $-e \mathbf{E}$ an electron between two successive collisions changes its velocity from $\mathbf{v}$ to $\mathbf{v}-e \mathbf{E} \tau / m$, where the mean time of the free flight, is

$$
\tau=\left(\frac{b n}{v^{3}}+\frac{v}{l}\right)^{-1}
$$

When $v$ corresponds to the thermal velocity $u$ the drift velocity of electrons obtained from $(31)$ is

$$
\mathbf{w} \approx-\frac{e \mathbf{E}}{2 m}\left(\frac{b n}{u^{3}}+\frac{u}{l}\right)^{-1} .
$$

(This almost coincides with Eq.(13) for $w_{z}$ ). In the stationary state the average energy gain between two collisions

$$
\Delta W=\frac{(e E \tau)^{2}}{2 m}
$$

should be equal to the average energy loss $(m / M) m\left(u^{2}-v_{i}^{2}\right)$,

$$
\left(\frac{e E}{m}\right)^{2}=\frac{2 m}{M}\left(u^{2}-v_{i}^{2}\right)\left(\frac{b n}{u^{3}}+\frac{u}{l}\right)^{2} .
$$

We see from $(33)$ that $u(E)$ is not a monotone function when $q=b n l / v_{i}^{4}$ is larger than about 12. This gives in a simplified form the origin of the kinetic transition. For this analysis to be reasonable the energies of the electrons must not be spread out too much, i.e. the bulk of electrons must have their speeds close to $u$. The e-e collisions provide the nonlinear cooperative coupling which brings about this condition. In the cylindrical plasma confined in a tube which was studied here the critical value of $q$ is found very close to 10 for plasma parameters used in Figs.1-3.

An S-shaped $T(E)$ or in other words a non-monotone behavior of the function $E(u)$ is caused by the decreasing term $b n / u^{3}$ in (33). The recombination of electrons near the tube walls makes the density $n$ a decreasing function of $u$, due to the growth of the charged particle mobility with temperature and therefore enhances the observation of our transition. The opposite role is played by the volume recombination whose rate decreases 
with the electron temperature ( $u$ here) and leads to a rise of density with $u$. We thus have a competition between these processes in the cylindrical geometry and may anticipate that the smaller is the bulk recombination the easier would be an experiment.

\section{Acknowledgments}

We thank Dr. Robert Barker both for useful specific comments and for general encouragement during the course of this work. We also thank Dr. Spencer Kuo for looking at possible experimental realizations of our kinetic transition and the referee for many helpful comments. Work supported by Air Force Office of Scientific Research Grant $01594-26435$. 


\section{Appendix: Nature of Approximations}

We rewrite now Eq.(2) in a dimensionless form in order to better see the mathematical nature of the approximations made in our work. Setting $\epsilon=\sqrt{m / M}, t^{\prime}=\epsilon t v_{i} / l, \mathbf{v}^{\prime}=$ $\mathbf{v} / v_{i}, \quad \mathbf{r}^{\prime}=\mathbf{r} / R, \epsilon \mathbf{w}^{\prime}=\mathbf{w} / v_{i}, \delta=l / R, \mathbf{E}^{\prime}=\left(e l / \epsilon m v_{i}^{2}\right) \mathbf{E}, \mathbf{F}^{\prime}=\left(e l / \epsilon m v_{i}^{2}\right) \mathbf{F}, \lambda=$ $l \sqrt{a c / v_{i}^{5}}, n\left(r^{\prime}\right)=n(r) \sqrt{c / a v_{i}^{3}}$, and dropping primes we get

$$
\begin{gathered}
\epsilon \frac{\partial f(\mathbf{r}, \mathbf{v}, t)}{\partial t}-\epsilon(\mathbf{E}+\mathbf{F}) \cdot \nabla_{\mathbf{v}} f+\delta\left(\mathbf{v} \cdot \nabla_{\mathbf{r}} f\right)= \\
\lambda\left(\psi-\frac{n}{x^{3}} f\right)+\epsilon^{2} \frac{1}{v^{2}} \frac{\partial}{\partial v}\left[\left(4 \omega n / 9+v^{4}\right)\left(\bar{f}+\frac{1}{3 v} \frac{\partial \bar{f}}{\partial v}\right)\right]+\frac{4 \omega n}{9 v^{3}} \hat{L} f+v(\bar{f}-f)+Q[f],
\end{gathered}
$$

where $\omega$ is defined in $(21)$ and we have

$$
\begin{gathered}
\int \psi d^{3} v=1, \quad \int \mathbf{v} \psi d^{3} v=0, \quad \int v^{2} \psi d^{3} v=\Theta \\
\int f d^{3} v=n(r), \quad \int \mathbf{v} f d^{3} v=\epsilon n(r) \mathbf{w}(r), \quad \int v^{2} f d^{3} v=n(r)\left[x^{2}(r)+\epsilon^{2} w^{2}(r)\right] .
\end{gathered}
$$

We restrict ourselves now to the homogeneous case $(R=\infty)$ with a fixed electron density, $n$, without ionization - recombination. Eq.(A1) then takes the form

$$
\frac{\partial f}{\partial \tau}-\mathbf{E} \cdot \nabla_{\mathbf{v}} f=\frac{\epsilon}{v^{2}} \frac{\partial}{\partial v}\left[\left(q+v^{4}\right)\left(\bar{f}+\frac{1}{3 v} \frac{\partial \bar{f}}{\partial v}\right)\right]+\frac{1}{\epsilon}\left\{\frac{q}{v^{3}} \hat{L} f+v(\bar{f}-f)+Q[f]\right\} .
$$

In the stationary state the equations for the moments have the form

$$
\begin{gathered}
\mathbf{E}+\frac{1}{\epsilon} \int \mathbf{v}\left(\frac{2 q}{v^{3}}+v\right) \Phi(\mathbf{v}) d^{3} v=0 \\
E w+\frac{4 \pi q}{3} \Phi(0)-\int\left(\frac{q}{v}+v^{3}-\frac{4 v}{3}\right) \Phi(\mathbf{v}) d^{3} v=0
\end{gathered}
$$

where $q=b n l / v_{i}^{4}$ as before and we have set $\Phi=f / n$ so that

$$
\int \mathbf{v} \Phi d^{3} v=\epsilon \mathbf{w}, \quad \int v^{2} \Phi d^{3} v=x^{2}+\epsilon^{2} w^{2}
$$

If one uses the substitution (7), which now has the form,

$$
\Phi(\mathbf{v}) \rightarrow(3 / 2 \pi)^{3 / 2} x^{-3} \exp \left[-\frac{3}{2 x^{2}}(\mathbf{v}-\epsilon \mathbf{w})^{2}\right]
$$

and neglects $\epsilon^{2}(w / x)^{2}$ compared to unity Eqs.(A3,A4) transform into the set

$$
E=\frac{2 w}{x^{3}} \sqrt{\frac{6}{\pi}}\left(q+4 x^{4} / 9\right)=0
$$




$$
E w=\sqrt{\frac{6}{\pi}} \frac{x^{2}-1}{x^{3}}\left(q+8 x^{4} / 9\right)=0 .
$$

In the stationary case $(\mathrm{A} 6, \mathrm{~A} 7)$ can be solved for $w(E)$ and $x(E)$ yielding non-unique solutions, when $q>7.6$ (see Ref.4). The solution of $(\mathrm{A} 6, \mathrm{~A} 7)$ for $x$ can be found from the relation

$$
E^{2}=\frac{12}{\pi} \frac{x^{2}-1}{x^{6}}\left(q+4 x^{4} / 9\right)\left(q+8 x^{4} / 9\right) .
$$

Eq.(A8) is close to Eq.(33), which was obtained in a rough approximation without referring to the kinetic equation.

It is natural to expand $f(\mathbf{v}, t)$ for the spatially homogeneous case in a series in $\epsilon$

$$
f(\mathbf{v}, t)=\sum_{j=0} f_{j}(\mathbf{v}, t) \epsilon^{j}
$$

Substituting (A9) into (A2) gives a set of coupled equations for $f_{j}$ where higher components can be expressed through lower ones. We do not use this method in the present work, instead we have solved Eq.(A1) using the method of moments with $\epsilon=1.4 \times 10^{-4}, \delta=$ $0.2, \lambda \approx 0.1$, and $\Theta=10$. In order to have a closed set of differential equations we have taken

$$
f_{1}=\left(\frac{3 \mathbf{v} \cdot \mathbf{w}}{x^{2}}\right) \bar{f}
$$

which comes from the shifted Maxwellian. We expect the effect of our additional approximation to be small. 


\section{REFERENCES}

[1] F.F.Chen, Introduction to Plasma Physics (Premium Press, New York, 1974), pp. 139-140, 158-160.

[2] V.A.Godyak, R.B.Piejak, and B.M.Alexandrovich, Plasma Sources Sci. Technol. 3, 169 (1994);

[3] IEEE Conference Record - Abstracts, 1995 IEEE International Conference on Plasma Science.

[4] A.V.Rokhlenko, Phys.Rev. A 43, 4438 (1991).

A.V.Rokhlenko and J.L.Lebowitz, Phys.Fluids B 5, 1766 (1993).

[5] E.Carlen, R.Esposito, J.L.Lebowitz, R.Marra, and A.Rokhlenko, Phys.Rev. E, 52, R40 (1995).

[6] R.N.Franklin, Plasma Phenomena in Gas Discharges (Clarendon Press, Oxford, 1976), pp.20-37;

[7] K.-B.Persson, Phys.Fluids 5, 1625 (1962).

D.B.Ilic, J.Appl.Phys. 44, 3993 (1973).

[8] H-B Valentini, J.Phys.D 21, 311 (1988).

[9] U.Kortshagen, Phys.Rev. E 49, 4369 (1994);

V.I.Kolobov and W.N.G.Hitchon, Phys.Rev. E 52, 972 (1995).

[10] V.E.Golant, A.P.Zhylinsky, and I.E.Sakharov, Fundamentals of Plasma Physics (Wiley, New York, 1980).

[11] R.Balescu, Transport Processes in Plasmas (North-Holland, Amsterdam-Oxford-New York-Tokyo, 1988), pp. 113, 138, 775-786.

[12] E.M.Lifshitz and L.P.Pitaevsky, Physical Kinetics (Pergamon, New York, 1981), pp. 93, 172, 181-184;

A.von Engel, Ionized Gases (AIP Press, New York, 1993), pp. 29-30, 243, 292;

C. Brown, Basic Data of Plasma Physics (AIP Press, New York, 1993).

[13] N.J.Carron, Phys.Rev. A 45, 2499 (1992).

[14] M.J.Druyvesteyn, Physica 10, 61 (1930);

M.J.Druyvesteyn and E.M.Penning, Rev.Mod.Phys. 12, 87 (1940).

[15] L.G.Christophorou, Electron-Molecule Interactions and Their Applications, vol.2 (Academic Press, Orlando, 1984), pp. 65-88. 
A.J.Cunningham and R.M.Hobson, Phys.Rev. 185, 98 (1969).

[16] R.Nagpal and A.Garscadden, Phys.Rev.Lett. 73, 1598 (1994).

[17] L.G.H. Huxley and R.W. Crompton, The Diffusion and Drift of Electrons in Gases (Wiley, New York, 1974), Chapter 14. 


\section{FIGURE CAPTIONS.}

Fig.1

The dependence of $x$ (representing the electrons thermal speed) and the current density on $\phi$ (the electric field squared) for the spatially homogeneous case $(R=\infty)$ when $\omega=250$. The dimensionless units are defined in Eq.(21) in the text.

\section{Fig.2}

The electron temperature $T$ and total current $I \cdot R$ (in $m A \cdot \mathrm{cm}$ ) vs the electric field $E \cdot R$ (in volts) when $T$ is assumed constant in the tube cross section, $T_{i}=300^{\circ}$ and $\omega=90$.

\section{Fig.3}

Plots of the mean electron temperature and total current when $T \not \equiv$ const and $\omega=90$. (The same units as in Fig.2). The hysteresis loop is indicated by arrows. At values of the field between the end points of the loop, like $E^{\prime}$, the computation leads to values on the lower or upper branches of the loop, determined by how close the starting point is to one of them.

\section{Fig.4}

The radial profiles of the mean electron speed and ambipolar electric field $F(r) \cdot R$ (in volts) when $\omega=90$ for two regimes: the solid lines correspond to a small field $\left(E \cdot R \approx 0.04 V, \bar{T} / T_{i} \approx 2.5\right)$, the dotted lines to a larger field $\left(E \cdot R \approx 0.07 V, \bar{T} / T_{i} \approx 8\right)$. 